\title{
Minimum Conflict Degree Channel Assignment in Multi-Rate Multi-Channel Wireless Networks
}

\author{
Dai-Wei Kuo, Chunhung Richard Lin, Ying Chih Lin \\ Dept. of Computer Science and Engineering \\ National Sun Yat-sen University \\ Kaohsiung 80424, Taiwan, R.O.C. \\ Email: david@mail.wtuc.edu.tw, lin@cse.nsysu.edu.tw, yclin@cse.nsysu.edu.tw
}

\begin{abstract}
In this paper, the conflict degree of multi-rate link pairs based on SINR model in co-channel has been analyzed. We suggest five conflict types for link pairs, and verify the associated effects on data rate links of each type in random topology network. To maximize the network throughput, we further propose a simple and efficient algorithm called Minimum Conflict Degree Channel Assignment (Min-CD CA). Novel metrics are also proposed to assess the conflict degree of each link and overall network. Our simulation results show that the proposed metrics are more appropriate than a widely used metric, fractional network interference, in multi-rate multi-channel wireless networks. The simulation results also show that our algorithm performs better than the minimum interference greedy CA algorithm.
\end{abstract}

Keywords-channel assignment; multi-channel; multi-rate; conflict; interference

\section{INTRODUCTION}

This is an important issue to maximize network throughput in IEEE 802.11 based wireless networks, and the transmission conflicts are major obstacles $[1,2]$. One type of conflicts is that the receiver is interfered by hidden transmitters. To avoid it, IEEE 802.11 MAC protocol adopts CSMA/CA scheme: a transmitter carrier senses channel before sending a packet, and proceeds if the channel is idle; otherwise, it waits for a while before next trial. However, it may lead to another kind of transmission conflicts because carrier sensing may hamper simultaneous transmissions. Several studies have been investigated to reduce these conflicts, and the common technique is the use of multiple channels [3-5].

IEEE 802.11a provides 12 (3 in b/g) orthogonal channels. Many channel assignment (CA) algorithms are proposed to minimize transmission conflicts. There are three main categories: static, dynamic, and hybrid, depending on the frequency with which the CA scheme is modified [5]. For the static scheme, the CA is constant, while it is frequently updated to improve the performance for the dynamic scheme. Between them, the hybrid scheme applies a static CA for some communication links and a dynamic one for others. Previous studies demonstrated that the channel switching cost in the dynamic CA could be in the order of milliseconds, implying that the static CA is more feasible for the commodity $\mathbf{8 0 2 . 1 1}$ hardware.
The total network interference, which is abbreviated as the conflict number in this paper, is extensively used to describe the conflict degree in multi-channel wireless networks [1,3]. In the paper, we find that the relations of carrier sensing and interference between link pairs have significant impacts on the throughput in multi-rate networks, and these effects shall not be ignored when trying to maximize network throughput.

Most CA schemes use the base rate for packet transmission without utilizing higher data rate communication links (H-links) effectively, and thus these schemes limit the network throughput improvement [7]. In IEEE $802.11 \mathrm{a} / \mathrm{b} / \mathrm{g}$ standards, multiple data rates are specified and $\mathrm{H}$-links usually require a higher signal to interference plus noise ratio (SINR) so that they can decode received data correctly. The transmission range of lower data rate links (L-links) is much larger than that of H-links. Therefore, the associated interference range of Llinks is also larger than that of $\mathrm{H}$-links [8]. We are interested in the conflict degree among these transmissions. We categorize five types of conflicts for link pairs. Accordingly, the distribution of different types of link pairs in a network is investigated and the impacts on throughput are explored. According to our analysis, we further propose an efficient algorithm for the static CA, named Minimum Conflict Degree Channel Assignment (Min-CD CA), to minimize the conflict degree of $\mathrm{H}$-links. The novel metrics are also provided to evaluate the conflict degree of each link and overall network. Compared with greedy approach, our simulation results show that the Min-CD CA algorithm is more efficient to enhance the network throughput in multi-rate multi-channel wireless networks.

This paper is organized as follows. We describe the motivating examples for our design of CA in section II, and review related works in section III. Section IV introduces the considered network model. In Section V, the conflict degree of link pairs is investigated and novel evaluation metrics are presented. Next, Section VI contains our algorithm Min-CD $\mathrm{CA}$, and we analyze the associated simulation results in Section VII. Finally, Section VIII draws our conclusions and future works.

\section{MOTIVATING EXAMPLES}

We consider a saturated traffic one-hop network and suppose all link pairs carrier sense with each other in Fig. 1. 


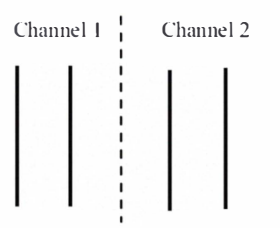

(a)

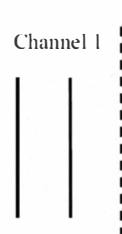$$
\text { (c) }
$$

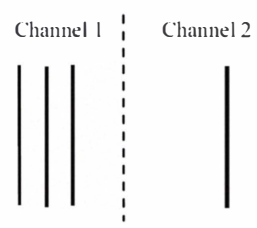

(b)

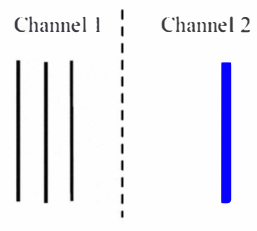

(d)

$$
\text { Chamnel } 2
$$

$6 \mathrm{Mbps}$
Figure 1. Difference of conflict number and conflict degree

According to the formulas in $[4,7]$, the network throughput is $2 /(1 / 6+1 / 6)+2 /(1 / 6+1 / 6)=12$ (Mbps) in Fig. 1(a) and the corresponding conflict number is $1+1=2$.

In Fig. 1(b), the network throughput is $[3 /(1 / 6+1 / 6+1 / 6)]+6$ $=12(\mathrm{Mbps})$ and its corresponding conflict number is $3+0=3$. They have the same network throughput although their conflict numbers are different. Furthermore, the network throughput is $2 /(1 / 6+1 / 6)+2 /(1 / 6+1 / 54)=16.8$ (Mbps) in Fig (c), and the corresponding conflict number is $1+1=2$. On the other hand, the network throughput is $[3 /(1 / 6+1 / 6+1 / 6)]+54=60$ (Mbps) in Fig. $1(\mathrm{~d})$, while its corresponding conflict number is $3+0=3$. The network throughput is larger when conflict number is larger. In Fig (c), the link pairs in channel 2 is called performance anomaly [9] since the throughput of the H-link is degraded below the data rate of the L-link. Obviously, the degree of conflict in H-link is greater than that of L-link. From above, the network with lower conflict number may not obtain a higher network throughput.

\section{RELATED WORKS}

In [2], the authors derived the upper bounds on the network throughput in wireless networks. Their works also explain why multiple channels can effectively increase the network throughput. In addition, many heuristics are proposed based on different constraints since the channel assignment problem is NP-hard [10]. Many studies alternatively adopted the simplified version of the interference mode, the fixed interference range model. In [11], the authors measured the effect of throughput under different relations of carrier sensing and interference for link pairs in an indoor 802.11a mesh network testbed, and suggested the SINR model [12] since the fixed interference range model is not correct in real world. In [13], the authors derived that the interference range is 1.78 times the distance between transmitter and receiver in the SINR model. In $[1,3,14]$, the authors minimized the conflict number to maximize the throughput in mesh networks. In the examples of Fig. 1, the network with a lower conflict number doesn't necessarily have a well throughput. The phenomenon could be more significant if the different conflict types of link pairs are considered in the multi-rate environment.

\section{NETWORK MODEL}

The RTS/CTS mechanism is shown to be ineffective and fails to increase the network throughput [13]. Therefore, we adopt the physical carrier sensing, whenever a wireless node intends to transmit, it first senses physically the medium. We adopt the SINR interference model [13], and assume that all the radio parameters are identical at each node and ignore the thermal noise. If $d_{t}$ is the distance between the transmitter and the receiver, the interference range $R_{i}$ of a signal at the receiver is given by $R_{i}=1.78 d_{t}$.

A network is modeled as a set of nodes (routers) and a set of wireless links. Each link supports two-way communication (data/ACK). A link exists between node $i$ and node $j$ if they are within the transmission range of each other and share a common channel. A link with transmitter $i$ and receiver $j$ is referred as $\operatorname{link}(i, j)$, and its data rate is denoted as $b_{i, j}$. In our model, we have the following assumptions. First, the number of radios of each router equals to its number of orthogonal channels. Second, our CA scheme is topology preserving, i.e., all links existing in a single channel network also exist in the multi-channel network after performing channel assignment. Third, we focus on the unicast saturated traffic load.

In this paper, the conflict means that transmitter can sense transmission from other nodes, or receiver is interfered by other transmitters. Compared with data packet, we ignore the interference caused by ACK packets because the associated packet size is very small.

\section{CONFLICT DEGREE OF LINK PAIRS}

\section{A. Conflict Types of Link Pairs}

There are several conflict types in multi-rate networks. According to different throughput of link pairs, they are categorized into five types.

In table I, only type 1 is conflict free. We simulate many combinations of multi-rate links in single channel with 30 nodes randomly distributed in a $1,000 \mathrm{~m} \times 1,000 \mathrm{~m}$ field. The data rates are 54, 18, 11, 6 and $1 \mathrm{Mbps,} \mathrm{where} \mathrm{their}$ corresponding transmission ranges are 76, 183, 304, 396, and $610 \mathrm{~m}$, respectively [15].

TABLE I. CONFLICT TYPES OF LINK PAIRS

\begin{tabular}{|c|l|c|}
\hline Type & $\begin{array}{c}\text { Relation of Carrier Sensing (CS) } \\
\text { and Interference }\end{array}$ & $\begin{array}{c}\text { Throughput of Link } \\
\text { Pairs }\end{array}$ \\
\hline 1 & no CS and no interference & $b_{i, j}+b_{m, n}$ \\
\hline 2 & $\begin{array}{l}\text { mutual CS and comparable data } \\
\text { rates }\end{array}$ & around $\operatorname{Max}\left(b_{i, j}, b_{m, n}\right)$ \\
\hline 3 & $\begin{array}{l}\text { mutual CS and links with } \\
\text { significant different data rates }\end{array}$ & around $\operatorname{Min}\left(b_{i, j}, b_{m, n}\right)$ \\
\hline 4 & no CS and one-way interference & $b_{i, j}$ or $b_{m, n}$ \\
\hline 5 & no CS and mutual interference & 0 \\
\hline
\end{tabular}



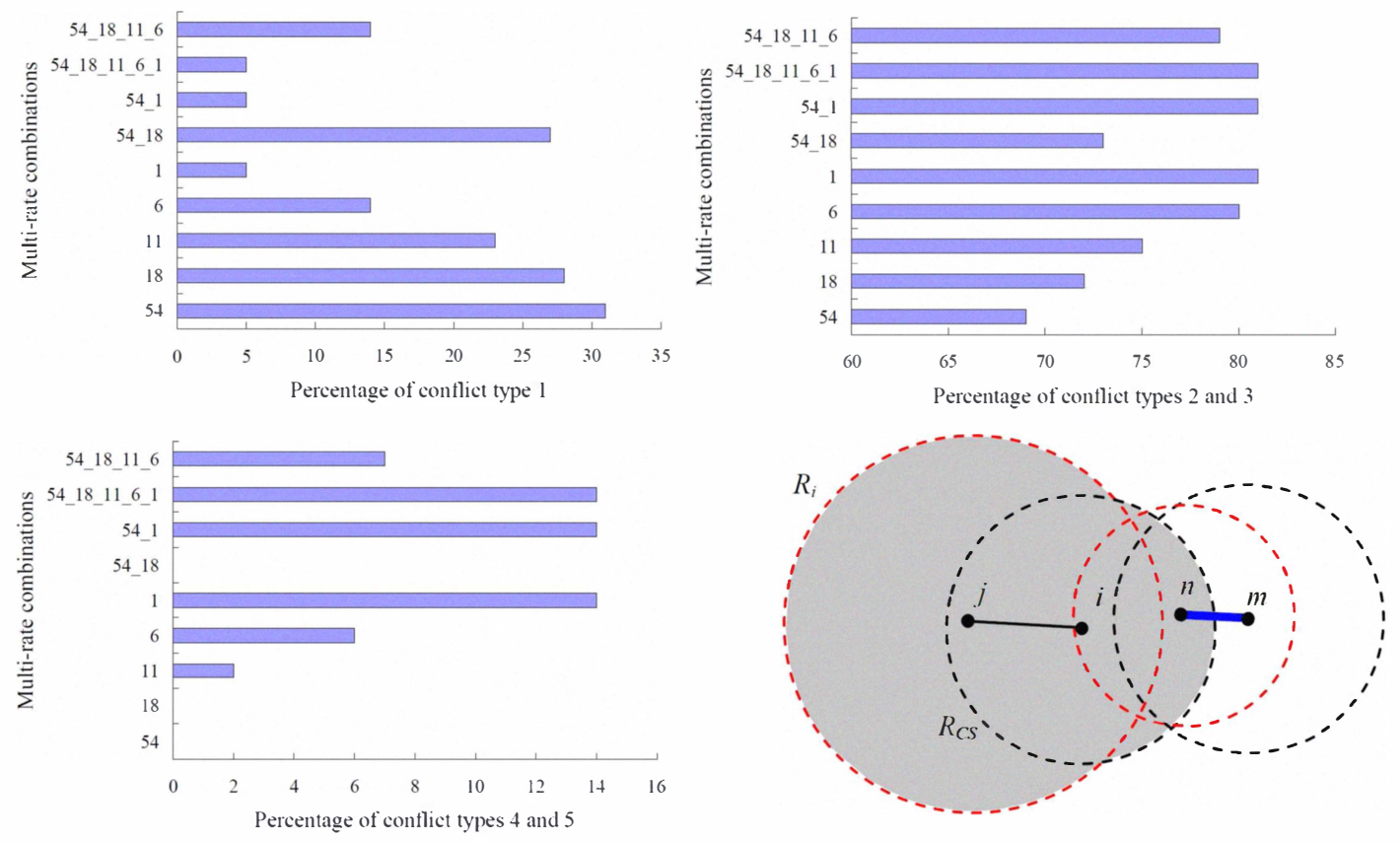

Figure 2. The conflict degree of link pairs in different multi-rate combinations

TABLE II. COMBINATION 54_18_11_6_1 IN SINGLE CHANNEL

\begin{tabular}{|c|c|c|c|}
\hline Data rate (Mbps) & Type 1 & Types 2 and 3 & Types 4 and 5 \\
\hline 54 & $15.4 \%$ & $67.2 \%$ & $17.4 \%$ \\
\hline 18 & $14.2 \%$ & $69.4 \%$ & $16.5 \%$ \\
\hline 11 & $7.9 \%$ & $81.7 \%$ & $10.5 \%$ \\
\hline 6 & $5.3 \%$ & $81.5 \%$ & $13.2 \%$ \\
\hline 1 & $1.3 \%$ & $80.2 \%$ & $18.5 \%$ \\
\hline
\end{tabular}

We use $610 \mathrm{~m}$ as physical carrier sensing range $\left(R_{C S}\right)$ and simulation results are shown in Fig. 2. From Fig. 2, when considering combinations of involving L-links, the percentage of conflict types 2-5 are more frequent than the conflict type 1 . Because the interference range of $54 \mathrm{Mbps}$ and $18 \mathrm{Mbps}$ is covered by carrier sensing range, they are not interfered. The transmission distance of L-links is larger than that of H-links, thus the interference range of L-links larger than that of $\mathrm{H}$-links. The conflict-zone of $\mathrm{L}-\operatorname{link}(i, j)$ is in grey as shown in Fig. 2. Since the interference range of L-link is larger than that of $\mathrm{H}$ link, the conflict-zone of L-links is larger than that of H-links. As a result, the conflicts in L-links occur more frequently than that in $\mathrm{H}$-links.

A link may involve multiple conflict types simultaneously with other links. We use combination of 54_18_11_6_1 scenario as an example to further analyze the percentage of different conflict types for various data rate links. Table II shows that 1 Mbps L-links have conflicts type 1 about $1 \%$. In other words, almost all the $1 \mathrm{Mbps}$ links have conflicts with other links.

Considering the multi-channel scenario, we assign $\mathrm{H}$ links(combination 54_18) and L-links(combination 11_6_1) to channel 1 and channel 2 , respectively. Compared with Table II, Tables III and IV show that the percentage of conflict type 1 in channel 1 significantly grows, but the variation is quite limited in channel 2. Hence, assigning H-link and L-links to different channels can greatly increase the percentage of conflict type 1 for $\mathrm{H}$-links, and subsequently, the network throughput is increased. But for L-links, the effect is not significant. Next, we assign the mixed combinations of $\mathrm{H}$-links and L-links (combinations 54_11_1 and 18_6) to channel 1 and channel 2, respectively. Tables $\bar{V}$ and $\overline{V I}$ show that the percentage of conflict type 1 for L-links slightly grows compared with that in table IV. But the percentage of conflict type 1 for $\mathrm{H}$-links is much smaller than that in Table III. Consequently, we shall assign $\mathrm{H}$-links and L-link respectively to different channels to increase the network throughput.

TABLE III. COMBINATION 54_18 In ChANNEL 1

\begin{tabular}{|c|c|c|c|}
\hline Data rate (Mbps) & Type 1 & Types 2 and 3 & Types 4 and 5 \\
\hline 54 & $36.5 \%$ & $63.5 \%$ & $0.0 \%$ \\
\hline 18 & $37.4 \%$ & $62.6 \%$ & $0.0 \%$ \\
\hline
\end{tabular}

TABLE IV. COMBINATION 11_6_1 In CHANNEL 2

\begin{tabular}{|c|c|c|c|}
\hline Data rate (Mbps) & Type 1 & Types 2 and 3 & Types 4 and 5 \\
\hline 11 & $10.8 \%$ & $75.9 \%$ & $13.2 \%$ \\
\hline 6 & $5.3 \%$ & $81.5 \%$ & $13.2 \%$ \\
\hline 1 & $1.3 \%$ & $80.2 \%$ & $18.5 \%$ \\
\hline
\end{tabular}

TABLE V. COMBINATION 54_11_1 In ChANNEL 1

\begin{tabular}{|c|c|c|c|}
\hline Data rate (Mbps) & Type 1 & Types 2 and 3 & Types 4 and 5 \\
\hline 54 & $15.4 \%$ & $67.2 \%$ & $17.4 \%$ \\
\hline 11 & $10.4 \%$ & $76.8 \%$ & $12.8 \%$ \\
\hline 1 & $2.2 \%$ & $80.5 \%$ & $17.3 \%$ \\
\hline
\end{tabular}

TABLE VI. COMBINATION 18_6 IN CHANNEL 2

\begin{tabular}{|c|c|c|c|}
\hline Data rate (Mbps) & Type 1 & Types 2 and 3 & Types 4 and 5 \\
\hline 18 & $26.4 \%$ & $68.7 \%$ & $4.9 \%$ \\
\hline 6 & $12.3 \%$ & $81.1 \%$ & $6.6 \%$ \\
\hline
\end{tabular}




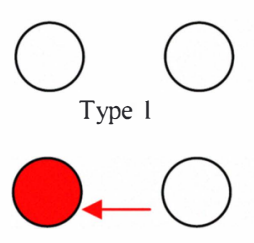

Type 4

(a) Conflict types of link pairs
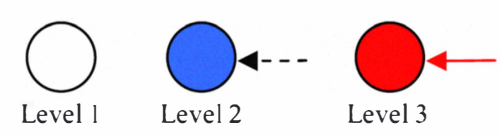

(b) Conflict levels of link in link pairs

Figure 3. Conflict types and conflict levels

\section{B. Novel Metrics of Conflict Degree for Link and Network}

There are three conflict levels defined for links. Level 1 is conflict free, and the throughput of link is equivalent to data rate of link (white nodes in Fig. 3). Level 2 means that a link senses other links but is not interfered, and hence it shares throughput with other links (blue nodes in Fig. 3). Level 3 means that a link is interfered by other links, indicating that the throughput is zero (red nodes in Fig. 3).

If a link has more level 1 relations with other links, the throughput of link is close to the data rate of link. There are more level 3 relations exist, the throughput of link is close to zero. In order to compare the performance of different CAs, we propose a novel metric to evaluate the potential conflict level of each link called Conflict Degree Index (CDI). If the CDI of link is close to one, the throughput of link is the data rate of link with higher probability. Otherwise, if it is close to zero, the throughput of link is zero with higher probability.

We define the $C D I_{i}^{k}$, which is the CDI of link $i$ in channel $k$, and the Potential Network Throughput Th $h_{\text {potential }}$ as follows:

$$
\begin{gathered}
C D I_{i}^{k}=\left(\sum_{j \in H_{i}^{k}} b_{i}+\sum_{j \in L_{i}^{k}} \frac{1}{b_{i}}+\frac{1}{b_{j}}\right) \times \frac{1}{\left(N_{k}-1\right) \times b_{i}} \\
T h_{\text {potential }}=\sum_{k=0}^{M-1} \sum_{i \in N_{k}} C D I_{i}^{k} \times b_{i}
\end{gathered}
$$

where $M$ is the number of non-overlapping channels in network, $N_{k}$ is the number of links in channel $k, b_{i}$ is the data rate of link $i, H_{i}^{k}$ is the level 1 link set of link $i$ in channel $k$, and $L_{i}^{k}$ is the level 2 link set of link $i$ in channel $k$.

When the link $i$ and link $j$ mutually sense each other in channel $k$, and $b_{i}$ is much greater than $b_{j}$ (for example, $b_{i}=54$ and $\left.b_{j}=1\right)$, the performance anomaly occurs. $C D I_{i}^{k}=[1 /(1 / 54$ $+1 / 1)] \times 1 / 54=1.8 \%$ and $C D I_{j}^{k}=[1 /(1 / 54+1 / 1)] \times 1 / 1=98.2 \%$. This means that if $\mathrm{H}$-link $i$ and L-link $j$ are active, then throughput of $\mathrm{H}$-link $i$ falls to $1.8 \%$ of $\mathrm{H}$-link $i$ data rate, and the throughput of L-link $j$ falls to $98.2 \%$ of L-link $j$ data rate.

\section{Minimum Conflict DEgREe CHANNEL ASSIGNMENT}

We propose a simple and efficient algorithm for the static CA, called Minimum Conflict Degree Channel Assignment (Min-CD CA). In our algorithm, we assign channels to links according to data rates and conflict types. The H-link is assigned to a channel with higher priority.

Suppose we have $M$ channels and $N$ links. Let Compare_Assign $(i, k)$ be the characteristic function for the existence of conflict type 1 when the link $i$ is compared with all links in channel $k$, i.e., Compare_Assign $(i, k)=$ TRUE if only conflict type 1 exists and FALSE otherwise. Moreover, if Compare_Assign $(i, k)$ returns TRUE, the link $i$ is assigned to channel $k$.

\section{Min-CD CA}

Initially, all links are ordered decreasingly according to data rates where the index is $0,1,2, \ldots, N-1$, and are assigned to channel 0 .

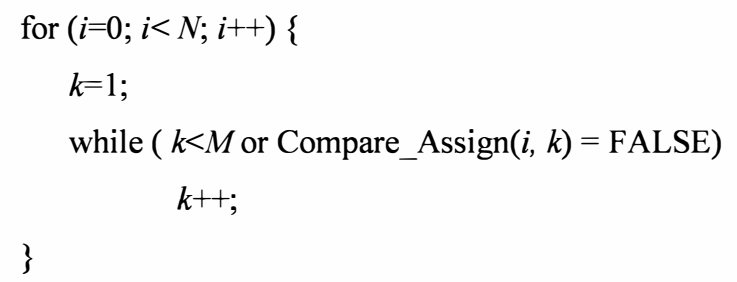

\section{SIMULATIONS}

In the simulations, we consider 10,20 , and 30 nodes randomly distributed over $1,000 \mathrm{~m} \times 1,000 \mathrm{~m}$ region. The transmission range is determined according to the literature [15]. We use data rates 54, 18, 11, $6 \mathrm{Mbps}$ in multi-rate case, and $6 \mathrm{Mbps}$ in base rate case. Physical carrier sensing range is $500 \mathrm{~m}$ and interference range is determined according to the SINR model. We simulate the random $\mathrm{CA}$, the minimuminterference (Min-I) greedy CA [1] and our proposed Min-CD CA. For the random CA, it allots channels randomly without further criteria. For the Min-I greedy CA, it greedily assigned channels so that the conflict number in overall network is minimized for each assignment trial. Two performance metrics are computed: Potential Network Throughput, which represents the potential network throughput of each CA. Fractional Network Interference, which is widely adopted in the minimum interference based CA and defined as the ratio of network conflict number after performing channel assignment relative to that in a single channel.

Fig. 4(a) and 4(b) demonstrate two performance metrics of three CAs in multi-rate and base rate scenarios, respectively. They show that the fractional network interference of Min-CD $\mathrm{CA}$ is larger than the other two CAs, but the associated potential network throughput is almost the same as the Min-I greedy. This implies that the fractional network interference and minimum interference based CA may be not appropriate for multi-rate multi-channel networks. Moreover, since the Min-I greedy CA is not a one-pass algorithm, our Min-CD CA is superior from the viewpoint of time complexity. The simulation result in Fig. 4(c) shows that the Min-CD CA outperforms the other two CAs. When more channels are 
available, this feature becomes more significant. The figures in Fig. 4 somehow point out that the key for assigning channels well is to consider the conflict number and the conflict degree together.

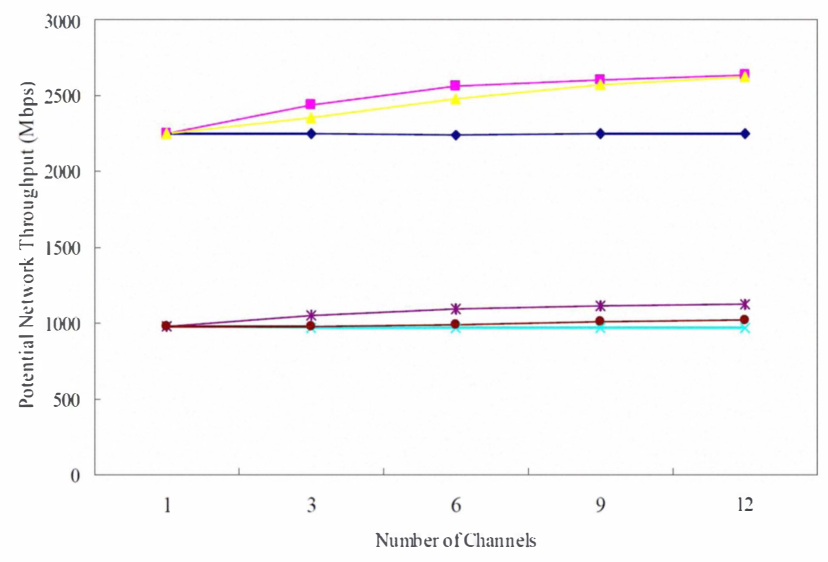

(a)

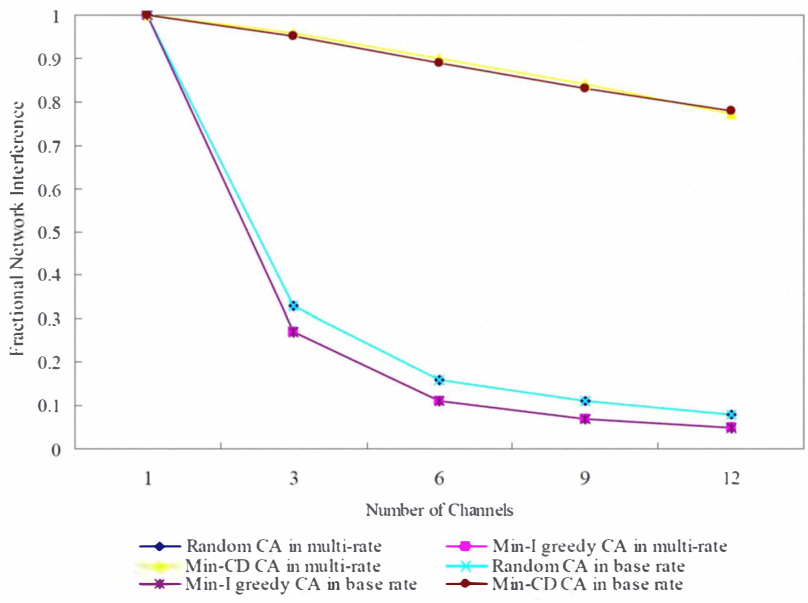

(b)

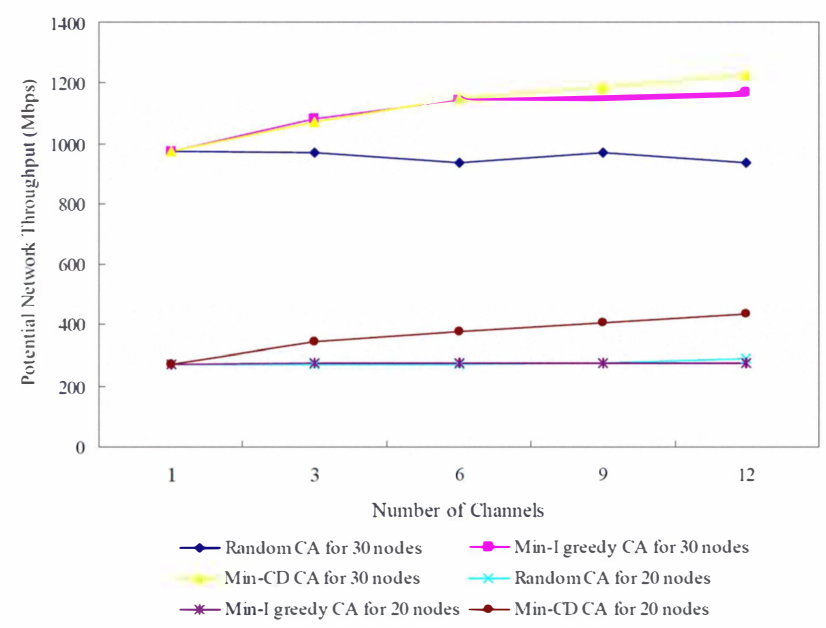

(c)

Figure 4. Comparison results of three CAs on two metrics

\section{CONCLUSIONS AND FUTURE WORKS}

In this paper, we propose a simple and efficient Min-CD $\mathrm{CA}$ algorithm to maximize network throughput. Novel metrics are also proposed to assess the conflict degree of each link and overall network. Our simulation results show that the proposed metrics are more appropriate than a widely used metric, fractional network interference, in multi-rate multi-channel wireless networks. The results also show that Min-CD CA performs well to improve network throughput. In the future, we are going to evaluate our proposed Min-CD CA and metrics regarding other traffic loads. The application of our proposed CDI metric in routing will be explored as well.

\section{REFERENCES}

[1] A.P. Subramanian, H. Gupta, S.R. Das, and C. Jing, "Minimum Interference Channel Assignment in Multiradio Wireless Mesh Networks," IEEE Transactions on Mobile Computing, vol. 7, no. 12, pp. 1459-1473, December 2008.

[2] P. Gupta and P.R. Kumar, "The Capacity of Wireless Networks," IEEE Transaction on Information Theory, vol. 46, no. 2, March 2000.

[3] M. Marina and S.R. Das, "A Topology Control Approach for Utilizing Multiple Channels in Multi-Radio Wireless Mesh Networks," 2nd International Conference on Broadband Networks, vol. 1, pp. 381-90, October 2005

[4] N. Niranjan, S. Pandeyand, and A. Ganz, "Design and evaluation of multichannel multirate wireless networks," Mobile Networks and Applications, vol. 11, pp. 697-709, October 2006.

[5] H. Skalli, S. Ghosh, S. K. Das, L. Lenzini, and M. Conti, "Channel assignment strategies for multiradio wireless mesh networks: Issues and solutions," IEEE Communications Magazine, vol. 45, pp. 86-95, November 2007

[6] L. Pan, S. Nicola, F. Yuguang, G. Enrico, and C. Imrich, "How to Effectively Use Multiple Channels in Wireless Mesh Networks," IEEE Transactions on Parallel and Distributed Systems, vol. 20, no. 11, pp. 1641-1652, November 2009.

[7] K. Lin and C. Chou, "Exploiting multiple rates to maximize the throughput of wireless mesh networks," IEEE Transactions on Wireless Communications, vol. 8, no. 12, pp. 6038-6049, December 2009.

[8] B. Alawieh, Z. Yongning, C. Assi, and H. Mouftah, "Improving Spatial Reuse in Multihop Wireless Networks - A Survey," IEEE Communications Surveys \& Tutorials, vol. 11, no. 3, pp. 71-91, 3rd Quarter 2009.

[9] M. Heusse, F. Rousseau, G. Berger-Sabbatel, and A. Duda, "Performance Anomaly of 802.11 b," Proc. INFOCOM'03, vol. 2, pp. 836-843, March 2003

[10] A. Raniwala, K. Gopalan, and T. Chiueh, "Centralized Channel Assignment and Routing Algorithms for Multichannel Wireless Mesh Networks," ACM SIGMOBILE Mobile Computing and Communications Review, vol. 8, pp. 50-65, April 2004.

[11] J. Lee, S.-J. Lee, W. Kim, D. Jo, T. Kwon, and Y. Choi. "Understanding interference and carrier sensing in wireless mesh networks, "IEEE Communications Magazine, vol. 47, pp. 102-109, July 2009.

[12] Karnik, A., Iyer, A., and Rosenberg, C., "What is the right model for wireless channel interference?," IEEE Transactions on Wireless Communications, vol. 8, no. 5, pp. 2662-2671, May 2009.

[13] K. Xu, M. Gerla, and S. Bae, "How effective is the IEEE 802.11 RTS/CTS handshake in ad hoc networks?," IEEE GlobeCom'02, vol. 1, pp. 72-76, November 2002

[14] J. Tang, G. Xue, and W. Zhang, "Interference-Aware Topology Control and QoS Routing in Multi-Channel Wireless Mesh Networks," ACM MobiHoc'05, May 2005.

[15] Cisco Aironet $802.11 \mathrm{a} / \mathrm{b} / \mathrm{g}$ Wireless LAN Client Adapters (CB21 AG and PI21AG) Installation and Configuration Guide. Cisco Systems, Inc., 2004. 